\title{
The Ability to Look: Management of Breast Disease in the Democratic Republic of the Congo Using Smart Ultrasound Technology
}

Ronda Henry-Tillman, MD, FACS, Mathew Kabongo, MD, Jonathan Laryea, MD, FACS, Leeya Pinder, MD, Rebecca Bittenbinder, MD, Geoffrey Osgood II, MD, Maya Hicks, BA, Michael Hicks, MD, Groesbeck Parham, MD

BACKGROUND: The vast majority of women with breast cancer in sub-Saharan Africa present with advanced stage disease, due primarily to the lack of opportunities for early detection and treatment. As part of a larger effort to increase access to diagnostic and therapeutic services for women's cancers in the Democratic Republic of Congo (DRC), we implemented a curriculum to train the local workforce and a program to build the supportive infrastructure for the diagnosis and treatment of breast cancer at a private sector health facility (Biamba Marie Mutombo Hospital) in Kinshasa.

STUDY DESIGN: After onsite trainings in the DRC by a US breast surgeon (RT), Congolese surgeons, general physicians, physician assistants, and nurses used the Phillips Lumify smart-phone ultrasound device to perform and interpret the results of whole breast ultrasound on symptomatic women. Surgeons were trained to perform ultrasound-guided core needle biopsy on those who met the criteria for tissue diagnosis, after which they trained nurses to do the same.

RESULTS: Over 3 years, 5,211 patients were identified as having a breast abnormality on clinical breast examination. Ultrasound abnormalities were noted in 1,493 (27\%) patients, of which 632 (42\%) met the criteria for ultrasound-guided core needle biopsy or fine needle aspiration. Pathology reports were available on 368 (58\%) patients who underwent biopsy, of which 164 were malignant and 204 benign.

CONCLUSIONS: We demonstrated how the "ability to look" using smart technology can be successfully used to augment clinical breast exam and triage patients for biopsy in a resource-constrained African setting. (J Am Coll Surg 2021;232:636-640. (C) 2020 by the American College of Surgeons. Published by Elsevier Inc. All rights reserved.)

Disclosure Information: Nothing to disclose.

Selected for the 2020 Southern Surgical Association Program.

Received December 1, 2020; Accepted December 1, 2020.

From the University of Arkansas for Medical Sciences, Department of Surgery, Winthrop P Rockefeller Cancer Institute, Little Rock, AR (Henry-Tillman, Laryea, Bittenbinder, Osgood II); Biamba Marie Mutombo Hospital, Democratic Republic of the Congo (Kabongo, Parham); University of Washington, Department of Oncology, Seattle, WA (Pinder); Michigan Cancer Institute, Pontiac, MI (Michael Hicks); University of North Carolina at Chapel Hill, Department of Obstetrics \& Gynecology, Chapel Hill, NC (Michael Hicks, Parham); Department of Obstetrics and Gynecology, University Teaching Hospital - Women and Newborn Hospital, Lusaka, Zambia (Michael Hicks, Parham); and Howard University College of Medicine, Washington, DC (Maya Hicks).

Correspondence address: Ronda Henry-Tillman, MD, FACS, University of Arkansas for Medical Sciences, Department of Surgery, Winthrop P Rockefeller Cancer Institute, 4301 West Markham, Slot \#725 Little Rock, AR 72205. email: henryrondas@uams.edu
Worldwide, breast cancer is diagnosed in more than 2 million women and is responsible for more than 521,000 deaths, annually. In Africa, it is the most commonly diagnosed cancer and the second leading cause of cancer-related death. ${ }^{1,2}$ It disproportionately affects young women in low to middle income countries (LMIC), with $23 \%$ of new cases occurring in women 15 to 49 years of age vs $10 \%$ in high-income countries. ${ }^{3}$ While screening mammography combined with high quality diagnostics and management (surgery, hormonal therapy, biologics, chemoradiation) have significantly improved disease outcomes in high income countries, such services are scarce in LMICs. ${ }^{4}$

In low-income settings, such as the DRC, the barriers to establishing women's care services are multifactorial. 


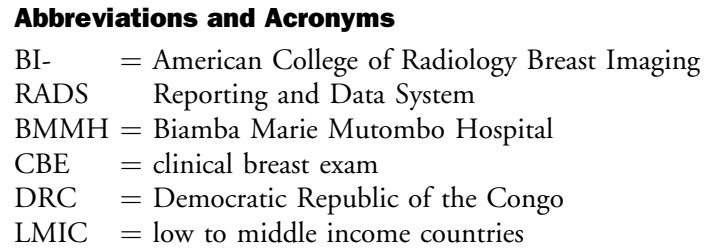

Some of these obstacles include, but are not limited to, underfunding, workforce shortages, and fragmented clinical infrastructure (eg ill-equipped healthcare facilities, unreliable supply chains, insufficient laboratory services, inadequate anesthesia support and blood banking capacity, lack of cancer data bases/registries, and weak systems for maintenance and repair). In such environments, ultrasound may be the best approach to breast evaluation given its relative lower costs, the fact that breast lesions and cancers in this population of women tend to occur earlier, when breasts are denser, and consensus guideline recommendations that breast masses should generally undergo diagnostic evaluation with ultrasound to distinguish their nature. ${ }^{5}$ Recent developments in ultrasound technology and portability, the incorporation of ultrasound training into the curriculum of healthcare workers caring for women with breast disease, and the use of percutaneous core biopsy device have all been essential in advancing the care of women who present with breast symptomatology. In this manuscript, we describe the use of ultrasound-based visualization of the breasts with portable smart technology in the DRC, how it was taught to healthcare providers, and its implementation to augment clinical breast exam (CBE) in symptomatic women.

\section{METHODS}

In 2017, a breast care clinic was established at the Biamba Marie Mutombo Hospital (BMMH), a private healthcare facility in the capital city of Kinshasa, in the Democratic Republic of Congo (DRC). Women screened for cervical cancer in a newly opened cervical cancer prevention clinic in the same facility were asked about breast symptoms and their willingness to have a CBE. Those answering in the affirmative underwent a $\mathrm{CBE}$ by trained Congolese cervical cancer screening nurses. If discovered to have abnormalities (masses, thickening, new onset asymmetry, pain, nipple discharge, axillary adenopathy, etc) they were referred to the newly established breast clinic for further evaluation with breast ultrasound. Ultrasound was performed with the Phillips Lumify app on a smart device (phone or tablet) with a specialized linear transducer, effectively converting the smart device into an ultrasound machine (Fig. 1). ${ }^{6}$ Women with suspicious lesions on ultrasound underwent ultrasound-guided core needle biopsy. All procedures were performed by Congolese healthcare workers, including nurses, physician assistants, and surgeons, initially under the on-site mentorship of a US breast surgeon (master trainer). Core biopsy specimens were sent to a private pathology laboratory for histologic examination. Trainee competency was evaluated using direct observation and concordance measurements between ultrasound impressions and pathologic diagnosis.

\section{Training}

In alignment with the approved curriculum, BMMH physicians, nurses, and physician assistants were trained in the following areas: breast self-awareness; CBE; smart phone and traditional breast ultrasound; axillary node ultrasound for the evaluation of suspicious axillary nodes; ultrasound-guided fine needle aspiration, and ultrasoundguided core needle biopsy of breast masses. The training was conducted by a senior US surgeon with expertise in ultrasound technology, teaching breast diagnostics, and surgical treatment of breast diseases. The curriculum included basic physics and principles of ultrasound. Detailed instructions and scanning techniques were performed on both live and phantom breast models. Detailed review consisted of instructions on recognition of the characteristics of ductal anatomy, normal breast parenchyma, benign vs malignant focal breast masses, and axillary nodal anatomy.

The American College of Radiology Breast Imaging Reporting and Data System (BI-RADS) was used as a part of the training curriculum. ${ }^{8}$ BI-RADS takes into account the shape of breast masses, their orientation, appearance of the margins, their echogenicity, posterior features, and other associated findings. All patients who underwent ultrasound and were found to have masses were evaluated using BI-RADS to help determine management. If an abnormality was solid with any indeterminate characteristic (taller than wide, lobulated borders, or septate), the patient was consented for a spring-loaded core biopsy. If a mass was anechoic with smooth borders, it was classified as cystic and aspirated. Lesions classified as BI-RADS 4 or 5 were biopsied, as were lesions classified as indeterminate.

Percutaneous core needle biopsy was demonstrated on phantom models, with emphasis on proper alignment of the linear transducer for biopsy. The hand-held, springloaded core biopsy device was placed in orientation with the transducer to allow the needle to be seen parallel to the transducer. Using sterile procedures, local anesthesia ( $1 \%$ lidocaine based on patient weight) was performed followed by a small puncture incision with a 


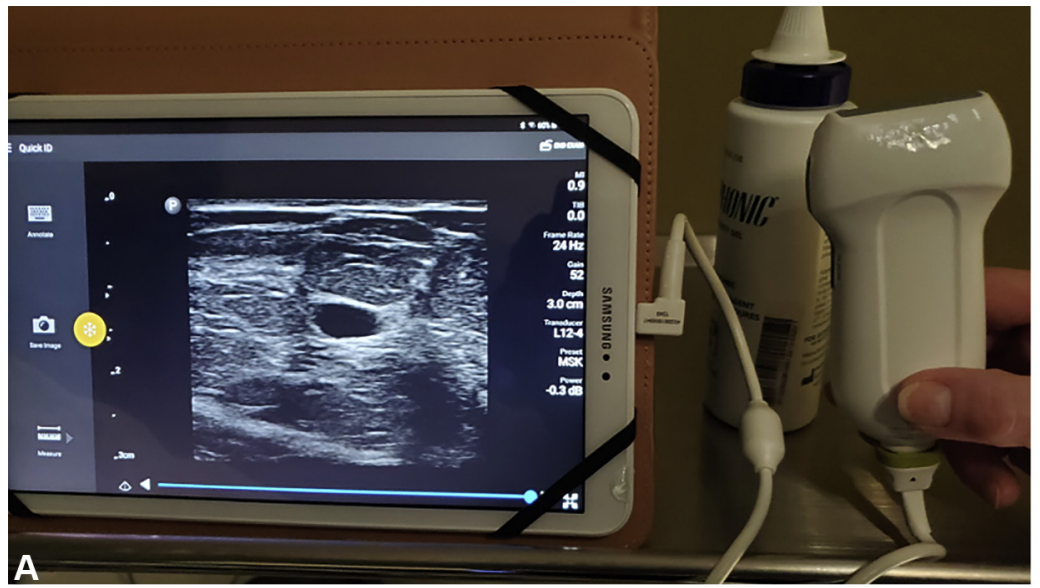

Figure 1. (A) Phillips Lumify Tele-Ultrasound Linear Ultrasound Transducer Probe; Ultrasound demonstrating image quality with both smart pad and smart phone.

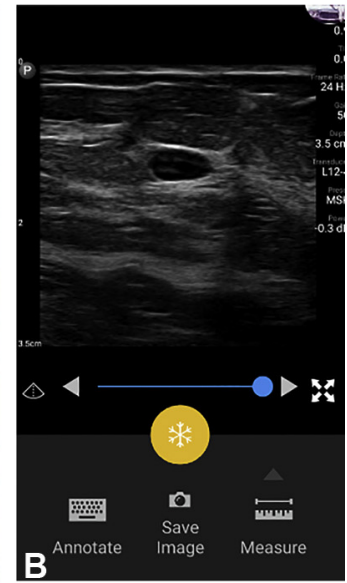

(B) Smartphone Enabled
\#11 scalpel blade. This was followed by the insertion of a $14 \mathrm{G}$ core needle (Fig. 2) into the breast under ultrasound guidance, after which 5 core biopsies were obtained.

After completion of the training program, the local clinical team was examined and checked off on the ability to demonstrate their understanding of the physics and principles of breast ultrasound and scanning techniques. This included recognition of normal and abnormal findings as well as demonstrating the ability to recognize and aspirate fluid collections (cyst or seroma) and how to perform a percutaneous biopsy using the spring-loaded device. Detailed review of pathology and its implications were evaluated and correlated with the clinical decisionmaking. Trainees were required to describe concordance and discordances in regard to ultrasound findings and

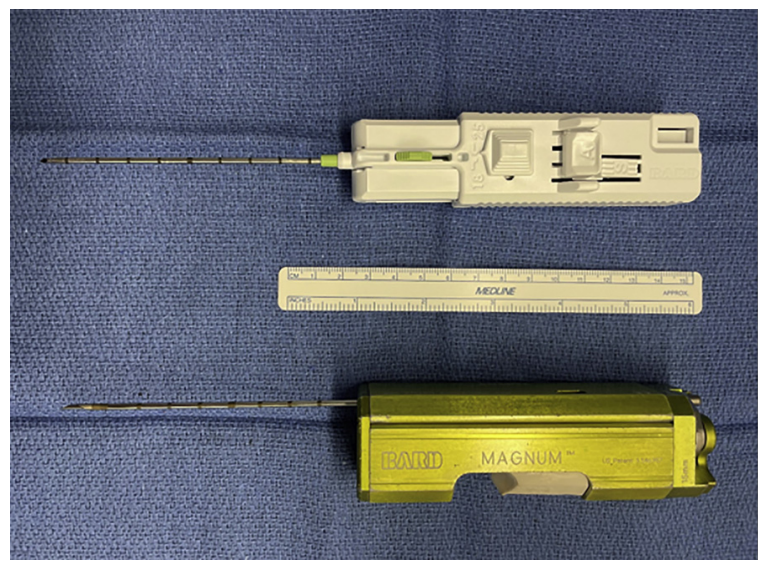

Figure 2. Disposable (top) and (bottom) reusable $14 \mathrm{G}$ percutaneous spring-loaded biopsy devices. pathologic results. Specific emphasis was placed on this understanding of concordance, defined as results of pathology consistent with interpretation of image findings. If core biopsy results were benign or malignant and ultrasound findings were benign or malignant, respectively, that was considered concordant. If cases of benign pathology in the face of suspicious ultrasound findings, trainees were instructed to perform an excisional biopsy.

\section{Trainee proficiency}

Data were collected and analyzed to assess the ability of the clinical team to effectively use the technology. This was performed using proctor assessments during quarterly visits of the master trainer, from 2017 to 2020 . Additional evaluations included self-assessments, how well trainees retained knowledge from quarter to quarter, how well the clinical team used the technology (Figs. 3, 4) and (Table 1), as indicated by number of ultrasounds performed, number of biopsies and descriptions of findings,

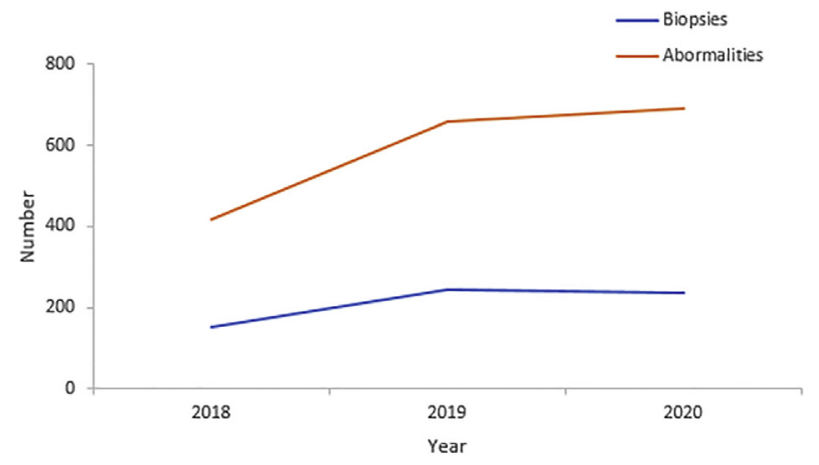

Figure 3. Use by of ultrasound and biopsy by year, 2018 to August 2020. 


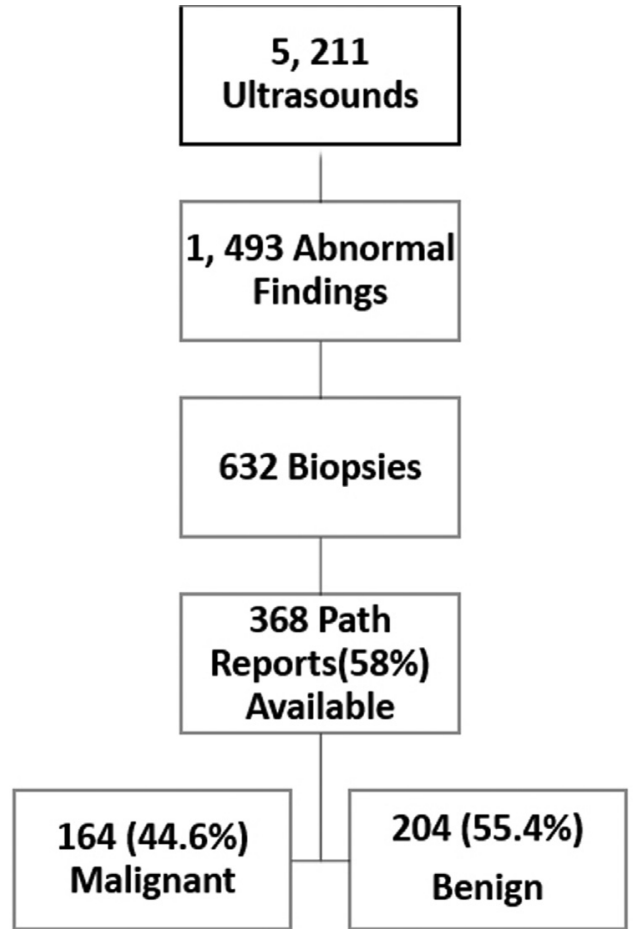

Figure 4. Data tree.

understanding the technical correlation, and concordance between ultrasound impressions and biopsy.

\section{RESULTS}

From April 2017 to August 2020, 5,211 patients were identified as having a breast abnormality on CBE. Ages ranged from 6 to 86 years; median age was 42.0 $( \pm 14.1)$ years. Ultrasound abnormalities were noted in $1,493(27 \%)$ patients, of whom $632(42 \%)$ met the criteria (indeterminate cystic lesion, solid or suspicious masses) for ultrasound-guided core needle biopsy or fine needle aspiration (Fig. 2). Pathology reports were available on $368(58 \%)$ of those who underwent biopsy, 164 of which were malignant and 204 benign (Fig. 4). The majority (approximately 90\%) of the cancers were advanced (TNM stages 3 and 4). The remainder were stage 2. Approximately half $(51 \%)$ of the malignancies were ductal carcinomas. The benign lesions were mostly

Table 1. Number of Biopsies and Abnormalities by Year

\begin{tabular}{lcc}
\hline Year & Biopsies, $\mathbf{n}$ & Abnormalities, $\mathbf{n}$ \\
\hline 2018 & 151 & 419 \\
\hline 2019 & 244 & 659 \\
\hline 2020 & 237 & 415 \\
\hline Total & 632 & 1,493 \\
\hline
\end{tabular}

Table 2. Concordance of Ultrasound with Pathology

\begin{tabular}{lccc}
\hline Variable & Ultrasound, $\mathbf{n}$ & Pathology, $\mathbf{n}$ & Concordance, $\%$ \\
\hline Benign & 141 & 141 & 100 \\
\hline Malignant & 91 & 94 & 97 \\
\hline
\end{tabular}

fibrocystic masses and fibroadenomas. Surgical procedures consisted of 183 lumpectomies, 58 modified radical mastectomies, and 45 axillary lymph node dissections. All cancer cases were referred to the newly developed chemotherapy unit at BMMH for evaluation. The concordance rate for ultrasound imaging and pathology was 97\% (91 of 94) for cancer and 100\% (141 of 141) for benign lesions (Table 2).

\section{DISCUSSION}

The ability to examine the breast using sound waves ("the ability to look"), then use the various findings to distinguish between normal breast tissue, common artifacts, and benign and malignant lesions is critical to appropriate decision-making, especially in highly resource-constrained environments, like the DRC, where pathology services are severely limited. This capability, combined with the new (smart device/phone or tablet apps), affordable (cost $<\$ 10,000$ ), and more portable (weight $<136$ grams) ultrasound devices makes it even more applicable as a tool in the clinical management of patients with breast disease in LMICs. As of 2020, almost $90 \%$ of all cell phones and smart devices on the African continent use the Android operating system. ${ }^{9}$ Therefore, for any smart technology to be of use in this region of the world, and similar environments, it should be capable of integration within this type of system.

Over time, as their clinical experience accumulated, the clinical team at BMMH gained greater competency using the ultrasound smart technology device, as demonstrated in Figs. 3 and 4. Clinical competency, defined as the identification of indicated lesions to be biopsied and the ability to safely and effectively perform core needle biopsies, was reached early in the training. Our concordance data (Table 2) of $97 \%$ for malignant and $100 \%$ for benign lesions are consistent with the data generally quoted in the literature. ${ }^{10}$ This is of utmost importance because it allows an accurate assessment without the cumbersome use of large, expensive, nonportable ultrasound equipment. It is also of great use in settings in which the "gold standard" of pathology is either nonexistent, unaffordable, or severely limited, and physicians are forced to rely on clinical impression alone to determine how breast lesions are managed.

The best chance for optimal outcomes in patients with breast cancer is early identification and diagnosis coupled 
with high quality treatment. In most LMICs, surgeons are not trained in ultrasound technology and therefore rely on radiologists for all diagnostic studies. The type of curriculum we implemented at BMMH allows the surgeon to become the primary diagnostician and interventionist, making the workup and treatment of breast diseases more timely and efficient. This is important in LMICs, where women sometimes have to travel long distances to reach healthcare facilities, and where it has been shown that delay in diagnosis is associated with worse outcomes. ${ }^{11,12}$

There were challenges in our program. First, the lack of in-house pathology services resulted in the use of outside private labs that were cost prohibitive to many patients. This led to 20 missing pathology reports. Second, the difficulty of procuring a steady supply of core needle biopsy guns into the country, for there was no suppler in the DRC; and third, the training literature for the Lumify App platform was not in the French language. BMMH has partnered with the American Society of Clinical Pathologist to develop a pathology laboratory at the hospital to overcome this impediment. We were able to find a local translator to translate the Lumify App instructions into French.

\section{CONCLUSIONS}

The "ability to look" into the substance of the breast using sound waves has many applications in the diagnosis and management of breast diseases, including cancer. Breast ultrasound, when used as an adjunct to physical examination, can be used to differentiate benign from malignant lesions and to allow for and direct clinical interventions. We combined its use with percutaneous core needle biopsy as the basic components of a platform for the evaluation symptomatic women in a resource-constrained environment. When integrated as a component of our innovative training curricula, it became a relevant training and patient management platform that is both affordable and scalable.

\section{Author Contributions}

Study conception and design: Henry-Tillman, Kabongo, Laryea, Pinder, Bittenbinder, Osgood II, Maya Hicks, Michael Hicks, Parham

Acquisition of data: Pinder, Kabongo, Maya Hicks

Analysis and interpretation of data: Henry-Tillman, Kabongo, Laryea, Pinder, Bittenbinder, Osgood II, Maya Hicks, Michael Hicks, Parham

Drafting of manuscript: Henry-Tillman, Kabongo, Laryea, Pinder, Bittenbinder, Osgood II, Maya Hicks, Michael Hicks, Parham
Critical revision: Henry-Tillman, Kabongo, Laryea, Pinder, Bittenbinder, Osgood II, Maya Hicks, Michael Hicks, Parham

Acknowledgment: The authors wish to acknowledge the Dikembo Mutombo Foundation, the Howard G Buffet Foundation, and the Friends of Africa, Inc.

\section{REFERENCES}

1. Ghoncheh M, Pournamdar Z, Salehiniya H. Incidence and mortality and epidemiology of breast cancer in the world. Asian Pac J Cancer Prev 2016;17[sup 3]:43-46.

2. Tfayli A, Temraz S, Mrad RA, Shamseddine A. Breast cancer in low- and middle-income countries: an emerging and challenging epidemic. J Oncol 2010;2010:1-5.

3. Freedman L, Barchana M, Al-Kayed S, et al. A comparison of population-based cancer incidence rates in Israel and Jordan. Eur J Cancer Prev 2003;12:359-365.

4. American Cancer Society. Breast cancer facts and figures. Available at: https://www.cancer.org/content/dam/cancer-org/research/ cancer-facts-and-statistics/breast-cancer-facts-and-figures/breastcancer-facts-and-figures-2019-2020.pdf. Accessed September 22, 2020.

5. Dempsey PJ. The history of breast ultrasound. J Ultrasound Med 2004;23:887-894.

6. Lumify transducers (probes) \& accessories: Philips Healthcare. Philips. Available at: https://www.usa.philips.com/healthcare/ sites/lumify/products-accessories. Accessed November 12, 2020.

7. Staren ED, Knudson MM, Rozycki GS, et al. An evaluation of the American College of Surgeons' ultrasound education program. Am J Surg 2006;191:489-496.

8. Mendelson EB, Böhm-Vélez M, Perf WA. ACR BI-RADS C Ultrasound In: ACR BI-RADSC Atlas, Breast Imaging Reporting and Data System 2013.

9. Mobile Operating System Market Share Africa. StatCounter Global Stats. Available at: https://gs.statcounter.com/osmarket-share/mobile/africa. Accessed November 30, 2020.

10. Youk JH, Kim E-K, Kim MJ, Oh KK. Sonographically guided 14gauge core needle biopsy of breast masses: a review of 2,420 cases with long-term follow-up. Am J Roentgenol 2008;190:202-207.

11. Foerster M, Mckenzie F, Zietsman A, et al. Dissecting the journey to breast cancer diagnosis in sub-Saharan Africa: Findings from the multicountry ABC-DO cohort study. Int J Cancer 2020;148:340-351.

12. Songiso M, Pinder LF, Munalula J, et al. Minimizing delays in the breast cancer pathway by integrating breast specialty care services at the primary health care level in Zambia. JCO Glob Oncol 2020;6:859-865.

\section{Invited Commentary}

\section{Suzanne Klimberg, MD, PhD, MSHCT, FACS Galveston, TX}

I enjoyed reading the Congo experience with ultrasound giving frontline practitioners the "ability to look." It certainly has increased the ability to find cancer in this relatively young 